\title{
The transition of an in-line vortex to slug flow: correlating pressure and reaction force measurements with high-speed video
}

\author{
B. J. de Witt \& R. J. Hugo \\ Department of Mechanical and Manufacturing Engineering, \\ University of Calgary, Calgary, Alberta, Canada
}

\begin{abstract}
In this work, the performance of a Slug Flow Expander (SFE) was investigated. Multiphase flow consisting of air and water was injected into the SFE. Critical flow rates of air and water were identified that caused transition to the slug flow regime. It was observed that at water flow rates of $1.00 \mathrm{~L} / \mathrm{s}$ and air flow rates above $3.00 \mathrm{~L} / \mathrm{s}$, the flow regime in the SFE transitioned to slug flow. A high-speed digital camera was used to visualize the flow inside the SFE. Wall pressure and reaction force measurements were synchronized with the high-speed camera. Autocorrelation, cross-correlation, and power spectral density functions were performed on reaction force, wall pressure, and air-core diameter. Power spectra of reaction force and wall pressure revealed strong energy peaks at low, middle, and high frequencies. Dominant low frequency energy $(2.9 \mathrm{~Hz}$ and $4.4 \mathrm{~Hz})$ was found to be attributed to the rotation rate of the liquid component in the SFE. The dominant middle frequency spikes $(39.5 \mathrm{~Hz}, 31.3 \mathrm{~Hz}, 24.4 \mathrm{~Hz}$ for reaction force; $20 \mathrm{~Hz}, 15.6 \mathrm{~Hz}$ for wall pressure) are believed to be attributed to the formation, convection, and exiting of the liquid slugs from the SFE. It is speculated that the dominant high-frequency spikes are attributed to both mechanical vibrations and pressure fluctuations caused by the flow facility pump.

Keywords: Slug Flow Expander, high-speed digital video, pressure and reaction force correlation, transition to slug flow regime.
\end{abstract}




\section{Introduction}

Many important industrial processes involve simultaneous flow of multiple phases that can include liquid, gas, and solids. A two-phase flow pattern called slug flow is encountered in horizontal pipe flow when a concentrated mass of liquid flows intermittently with a gas phase over certain ranges of flow rates. In a vertically oriented pipe, slug flow occurs when a sequence of liquid slugs successively follow long bubbles [1]. Understanding slug flow is made difficult by the multidimensional fluid dynamic processes that govern it.

An innovative mechanism by which slug flow can be initiated involves a Slug Flow Expander (SFE), shown in Fig. 1(a). Based on previous experimental results, slug flow in a vertical section of pipe was initiated by introducing liquid tangentially into a cylindrical cavity, causing a swirl flow to develop [3-5]. This results in annular swirl flow, with the central air-core surrounded by a liquid coaxial shell. When the air flow rate is increased, the diameter of the aircore decreases, resulting in the amplification of liquid-gas instability waves that eventually grow to subtend the entire pipe. This is shown in section $D-D$ ' in Fig. 1(b). Collapse of the air-core initiates the slug flow regime.

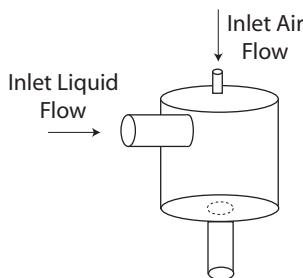

(a)

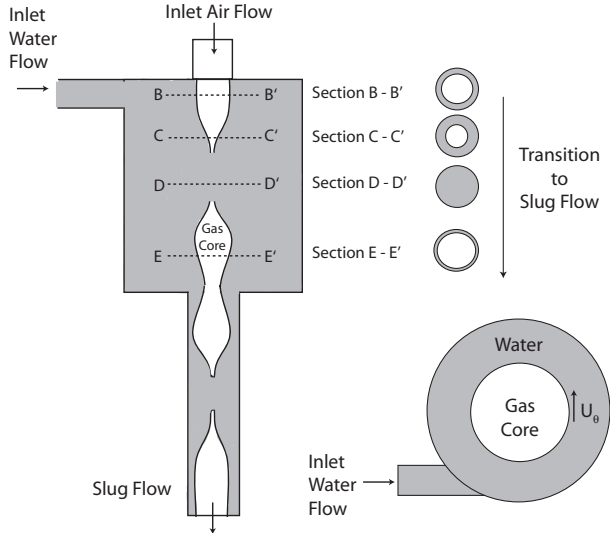

(b)

Figure 1: Slug Flow Expander (SFE): (a) 3-D rendering of SFE, (b) transition to the slug flow regime.

The central focus of this investigation was to study the transition to the slug flow regime and to investigate flow conditions that impart maximum momentum to the liquid. Flow rates of water and air that maintained a stable slug flow regime within the SFE were identified. High-speed video was then used to correlate pressure fluctuations inside the SFE cavity with the reaction force caused by accelerating liquid slugs on the entire mechanical unit. The paper begins with 
a description of the experimental apparatus used to complete the investigation, followed by a section that discusses high-speed video data synchronized with pressure fluctuations inside the SFE cavity and reaction force of liquid slugs. The next section performs statistical analysis of reaction force, wall pressure, and aircore diameter data. A conclusions section follows.

\section{Experimental apparatus}

The experimental apparatus consisted of two main parts: (1) the Slug Flow Expander with associated flow piping and (2) a high-speed digital video camera.

\subsection{SFE}

Figure 2 depicts the design of the SFE section. It was similar to models used by previous researchers [2-5, 7]. It included a tangential inlet tube, cylindrical cavity, end caps, and a downstream tube. The inlet tube was constructed of cast acrylic tubing. This section introduced water tangentially into the cylindrical cavity, inducing a swirl component. Transparent plexiglass sheets were used for both the top and bottom end caps of the cylindrical cavity, with a $\frac{1}{4}^{\prime \prime}(6.35 \mathrm{~mm})$ NPT hole tapped at the center of the top end cap to allow air to flow into the cylindrical cavity. Figure 2 also illustrates a wall pressure port, allowing static pressure measurements to be made near the inside wall.

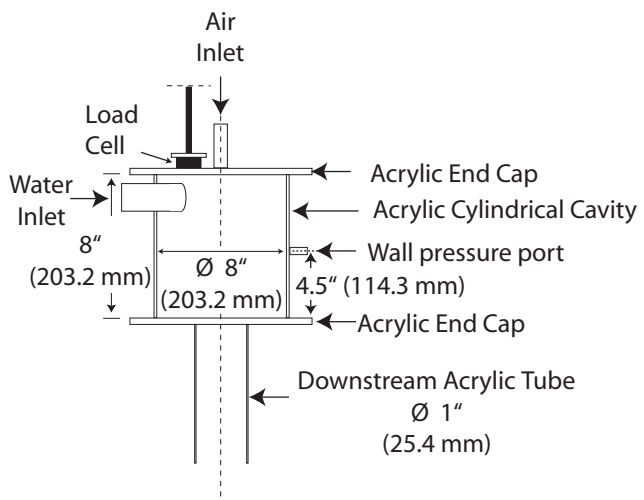

Figure 2: Schematic of the SFE.

\subsection{Instrumentation}

There were several measurement devices used in this experiment. The components included a Photron Ultima 1024 Digital Camera, a pressure transducer that monitored wall pressure, and a load cell that monitored reaction force of the entire SFE unit. 


\subsubsection{High-speed digital camera}

A high-speed digital camera (Photron-Ultima 1024) was used to interrogate the flow inside the SFE. Images were acquired at $1000 \mathrm{fps}$ at $512 \times 1024$ resolution. The analog voltage output from the wall pressure and reaction force transducers were synchronized with the high-speed camera using a Multi-Channel-Data-Link (MCDL). The A/D conversion resolution of the MCDL was 11 bits, with a sampling rate of $10 \mathrm{kHz}$. A total of $1.024 \mathrm{~s}$ of high-speed video and MCDL data was acquired for each flow condition.

\subsubsection{Pressure measurement}

A Validyne DP15 differential pressure transducer was used to perform pressure fluctuation measurements close to the wall of the cylindrical cavity. The Validyne DP15 had a frequency response of $1 \mathrm{kHz}$ and featured interchangeable diaphragms enabling the range of the transducer to be adjusted. The material connecting the transducer to the SFE was $\frac{1}{4}^{\prime \prime}(6.35 \mathrm{~mm})$ Nyaflow tubing, a semi-rigid plastic material. This reduced the amount of mechanical vibration transferred to the pressure transducer, while not degrading the pressure signal through the expansion and contraction of the tubing.

\subsubsection{Reaction force measurement}

The reaction force of the entire SFE unit was monitored using an OMEGA DLC101 Dynamic Force Sensor (load cell). During experiments, the load cell was kept in slight compression with a $\frac{1}{4}^{\prime \prime}(6.35 \mathrm{~mm})$ steel bolt. The load cell had a frequency response from $0.08 \mathrm{~Hz}$ to $25 \mathrm{kHz}$, calibrated by the manufacturer.

\section{High-speed camera measurements and results}

For the data presented in this work, the water flow rate was fixed at $1.0 \mathrm{~L} / \mathrm{s}$ and the air flow rate was varied from $0 \mathrm{~L} / \mathrm{s}$ to $8.0 \mathrm{~L} / \mathrm{s}$. High-speed video data was collected for water flow rates of $1.0 \mathrm{~L} / \mathrm{s}$ with varying flow rates of air. Air flow rates varied from $0 \mathrm{~L} / \mathrm{s}, 1.25 \mathrm{~L} / \mathrm{s}, 4.0 \mathrm{~L} / \mathrm{s}$, and $8.0 \mathrm{~L} / \mathrm{s}$. Additionally, a PC with a PowerDAQ PD2MF 16-333/16H data acquisition card was used to collect $30 \mathrm{~s}$ of data (sampling at $1 \mathrm{kHz}$ ) for wall pressure, and reaction force fluctuations for each component flow rate. This card had a 16-bit A/D converter capable of sampling 16 channels. This additional data set supplemented the $1.024 \mathrm{~s}$ of data collected using the MCDL, enabling better statistical representation. All data collected in this investigation were sent through low-pass anti-alias filters set to reject frequency components above $100 \mathrm{~Hz}$. Three different flow regimes were observed: (1) annular swirl flow (ASF) regime; (2) transition to slug flow (T) regime; and (3) slug flow (SF) regime. 


\subsection{Flow regimes observed in SFE}

The ASF regime was observed at water flow rates of $1.00 \mathrm{~L} / \mathrm{s}$ (air flow $0 \mathrm{~L} / \mathrm{s}$ ), as shown in Fig. 3(a). This flow regime was characterized by an air-core surrounded by an annulus of liquid in the larger cylindrical cavity. In the downstream acrylic tube, the flow appeared as a liquid film coating the outer edges of the acrylic tube. The high-speed video data set for this water flow rate $(1.00 \mathrm{~L} / \mathrm{s})$ was comprised of 1024 images. Figure 3(a) shows frame 100 out of 1024 images. Reaction force and wall pressure measurements for the $1.024 \mathrm{~s}$ of data are also shown.

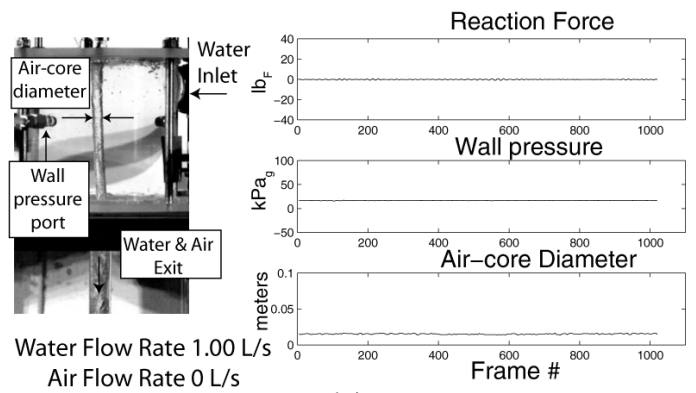

(a)
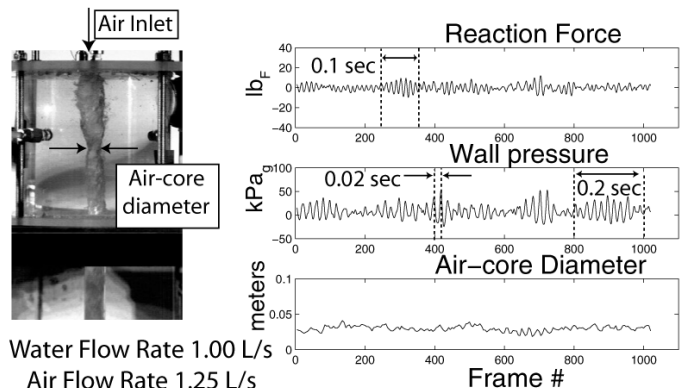

(b)

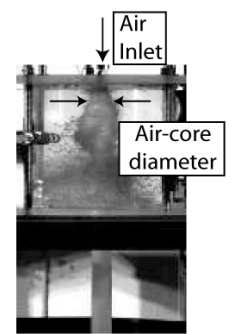

Water Flow Rate $1.00 \mathrm{~L} / \mathrm{s}$ Air Flow Rate $8.00 \mathrm{~L} / \mathrm{s}$

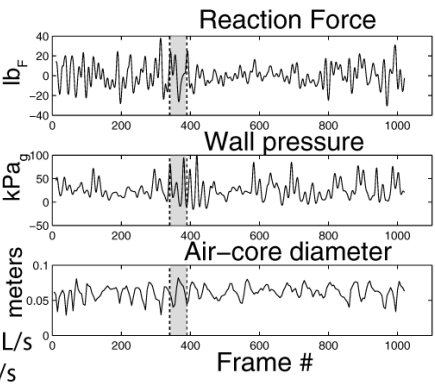

(c)

Figure 3: High-speed video data. (a) ASF, (b) T, and (c) SF regimes.

The $\mathrm{T}$ flow regime was observed when injecting air into the SFE at 1.25 $\mathrm{L} / \mathrm{s}$, as shown in Fig. 3(b). In this flow regime the diameter of the air-core was 
observed to vary in size, but did not fully collapse. Reaction force and wall pressure fluctuations are displayed in Fig. 3(b), illustrating the instabilities of this flow regime. The time-history for reaction force exhibited a high-frequency oscillation of approximately $70 \mathrm{~Hz}(0.014 \mathrm{~s})$, and low-frequency oscillation of 10 $\mathrm{Hz}$. The time-history for the wall pressure exhibited a high-frequency oscillation of approximately $50 \mathrm{~Hz}(0.02 \mathrm{~s})$, and a low-frequency ("beat") oscillation of $5 \mathrm{~Hz}$ (0.2 s). Also included in Figs. 3(a) and 3(b) are the diameters of the air-core for each frame, and this was measured by importing images into Adobe Photoshop. The number of pixels spanning the air-liquid interface at the axial location of the wall pressure port was calibrated to a known length in the image. In the ASF regime, the air-core diameter remained a constant size. In the $\mathrm{T}$ flow regime, the diameter of the air-core was shown to vary in size.

Slug flow, shown in Fig. 3(c) was observed for water flow rates of $1.00 \mathrm{~L} / \mathrm{s}$ combined with air flow rates above $3.00 \mathrm{~L} / \mathrm{s}$. The SF regime was characterized by large concentrated masses of liquid accelerating through the SFE at regular intervals. The air-core would collapse at regular intervals, and the SFE would shake violently due to the accelerating liquid slugs. Figure 3(c) was frame 639 out of this data set. Reaction force and wall pressure are shown accompanying Fig. 3(c). These measurements were more pronounced in the SF regime when compared to the ASF and T flow regimes. The dotted vertical lines designate a region of interest where a liquid slug was observed to develop, shown in greater detail within Fig. 4. Figure 4(a) shows 6 images, with wall pressure and reaction force displayed above each frame. Figure 4(b) displays accompanying reaction force, wall pressure and air-core diameter measurements.

The wall pressure of the cylindrical cavity was observed to rise due to the following: (1) localized pressure events triggered by liquid slugs passing by the wall pressure port and (2) large-scale pressure events triggered by liquid slugs exiting from the cylindrical cavity. Frame \#349 in Fig. 4(a) illustrates the beginning of the slug formation, marked by the sudden "necking" of the air-core diameter near the top acrylic end-cap. This "necking" was shown to accelerate downward in Frame \#355. Wall pressure continued to rise to $38 \mathrm{kPa}_{\mathrm{g}}$ in Frame \#361. Frame \#374 featured two events: (1) a liquid slug being purged from the cylindrical cavity into the downstream tube and (2) "necking" near the top acrylic end-cap. The wall pressure dropped to $4.7 \mathrm{kPa}_{\mathrm{g}}$ in this frame, but increased significantly to $94 \mathrm{kPa}_{\mathrm{g}}$ in Frame \#380 as the liquid slug exited the cavity. When examining the 30 second time history for wall pressure, the high-frequency pressure "spikes" were associated with liquid slugs exiting the cylindrical cavity. Frame \#385 illustrates the liquid slug completely exiting the SFE unit and the "necking" of the air-core diameter near the acrylic end-cap.

The diameter of the air-core for each frame is shown in Fig. 4(b). From the highspeed video, the air-core was found to flatten and stretch in the slug flow regime. There was uncertainty in this measurement because it was assumed the air-core was cylindrical. 


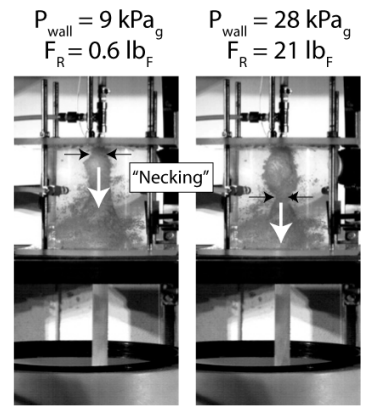

Frame 349
Frame 355

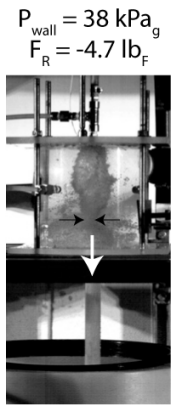

Frame 361

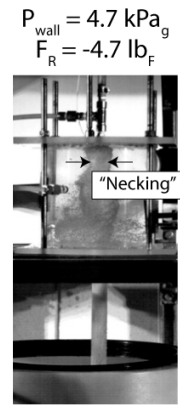

Frame 374

(a)
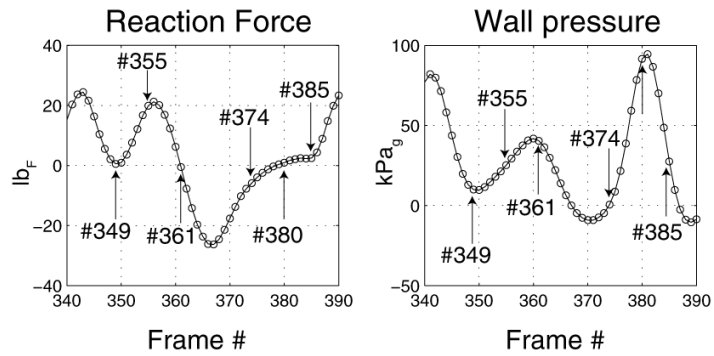

(b)

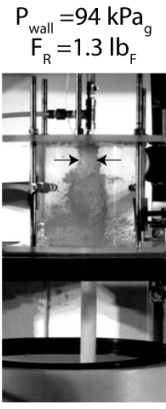

Frame 380
$P_{\text {wall }}=17 \mathrm{kPa}_{\mathrm{g}}$ $\mathrm{F}_{\mathrm{R}}=3.2 \mathrm{lb}_{\mathrm{F}}$

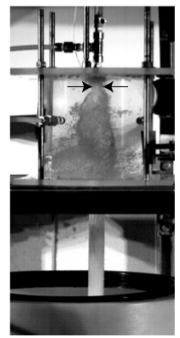

Frame 385

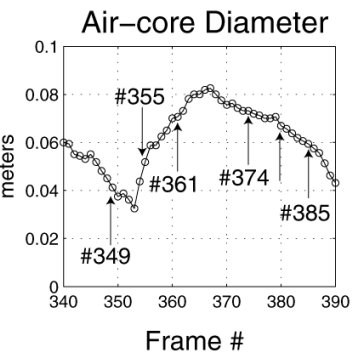

Figure 4: High-speed video data - slug flow (SF) regime: (a) image sequence, (b) reaction force, wall pressure, air-core diameter - frames \#340 to \#390 of data set shown in Fig. 3(c).

\subsection{Data analysis}

Correlation between reaction force, wall pressure, and air-core diameter is examined in greater detail in this subsection. The aforementioned signals are analyzed by computing autocorrelation coefficient , cross-correlation coefficient, and power spectral density functions.

The $30 \mathrm{~s}$ of data described at the beginning of Section 3 was used to calculate autocorrelation coefficient functions for reaction force and wall pressure. They are shown in Figs. 5(a) and 5(b). Examining autocorrelation coefficients of reaction force for air flow rates of $0 \mathrm{~L} / \mathrm{s}$, a middle-frequency component of $0.016 \mathrm{~s}(62.5$ $\mathrm{Hz}$ ) was discovered. The wall pressure autocorrelation revealed a high-frequency oscillation of $0.011 \mathrm{~s}(91 \mathrm{~Hz})$. For air flow rates of $1.25 \mathrm{~L} / \mathrm{s}$, frequency oscillations of $0.014 \mathrm{~s}(71 \mathrm{~Hz})$ and $0.012 \mathrm{~s}(83 \mathrm{~Hz})$ were resolved for reaction force and wall pressure, respectively. For air flow rates of $4.00 \mathrm{~L} / \mathrm{s}$ frequency components were present for reaction force and wall pressure at $0.014 \mathrm{~s}(71 \mathrm{~Hz})$ and $0.011 \mathrm{~s}(91 \mathrm{~Hz})$, respectively. Low-frequency ("beat") oscillations were measured at $0.306 \mathrm{~s}$ (3.26 $\mathrm{Hz}$ ) for both reaction force and wall pressure. This "beat" oscillation shifted to lower frequencies as air flow was increased to $8.0 \mathrm{~L} / \mathrm{s}$. Reaction force experienced a "beat" of $0.313 \mathrm{~s}(3.19 \mathrm{~Hz})$, while wall pressure revealed these effects at $0.320 \mathrm{~s}$ 
$(3.125 \mathrm{~Hz})$. This "beat" frequency was attributed to the angular rotation rate of the liquid component in the SFE.
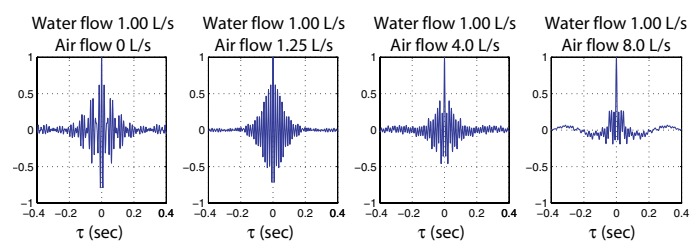

(a)
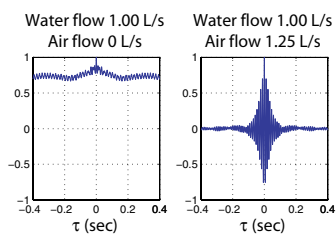

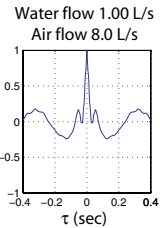

(b)

Figure 5: Auto-correlation coefficients: (a) reaction force, (b) wall pressure.

Cross-correlation coefficient functions were computed for the following: (1) wall pressure and reaction force; and (2) wall pressure and air-core diameter. The results displayed in Fig. 6(a) were for wall pressure and reaction force, using the $30 \mathrm{~s}$ of data described at the beginning of Section 3. Cross-correlation coefficient functions between wall pressure and reaction force were shown to be below 0.4 for air flow rates of $0 \mathrm{~L} / \mathrm{s}$ and $1.25 \mathrm{~L} / \mathrm{s}$. As the air flow rate was increased to 4.00 $\mathrm{L} / \mathrm{s}$, cross-correlation coefficients peaked to 0.1 , signifying that the two signals were somewhat correlated. When air flow rates of $8.00 \mathrm{~L} / \mathrm{s}$ were injected into the SFE, cross-correlation coefficients were calculated to be 0.4 , indicating that wall pressure and reaction force signals were well correlated and in phase. Figure 6(b) shows cross-correlation coefficients for wall pressure and air-core diameter, using only the $1.024 \mathrm{~s}$ of data synchronized with high-speed video. Cross-correlation coefficients for air flow rates of $0 \mathrm{~L} / \mathrm{s}$ and $1.25 \mathrm{~L} / \mathrm{s}$ signified nearly no correlation. Cross-correlation coefficients for air flow rates of $4.00 \mathrm{~L} / \mathrm{s}$ and $8.0 \mathrm{~L} / \mathrm{s}$ show that wall pressure and air-core diameter are slightly out of phase.

By computing power spectral density (PSD) functions of reaction force and wall pressure signals, dominant spectral components were identified. Power spectral density functions of wall pressure and reaction force signals were estimated using Welch's method [6], based on a 2048 point FFT using a Hanning window and $50 \%$ data overlap. Power spectral density function estimates are referred to as "wall pressure spectra" and "reaction force spectra," respectively. Figure 7 shows wall pressure spectra and reaction force spectra. These spectra are shown with increasing air flow rate, shifted incrementally in the vertical direction. Dominant spectral components at low, middle, and high frequencies are indicated in Figs. 7(a) and $7(\mathrm{~b})$. 

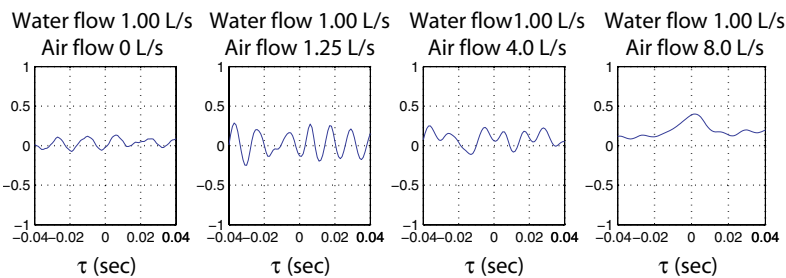

(a)
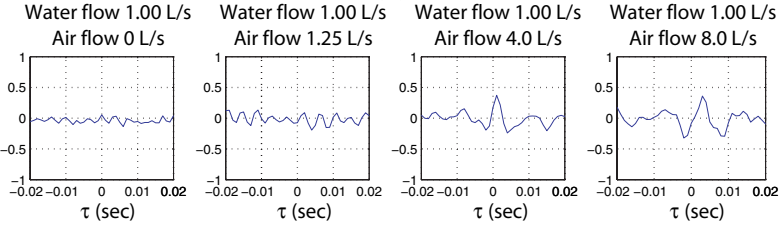

(b)

Figure 6: Cross-correlation coefficients (SF regime): (a) wall pressure and reaction force, (b) wall pressure and air-core diameter.

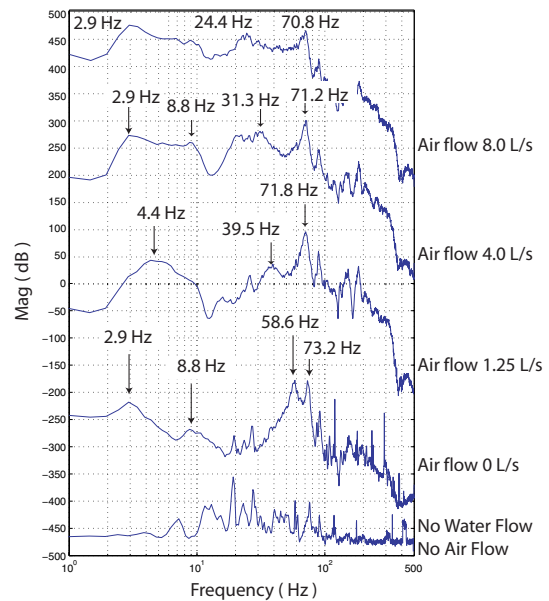

(a)

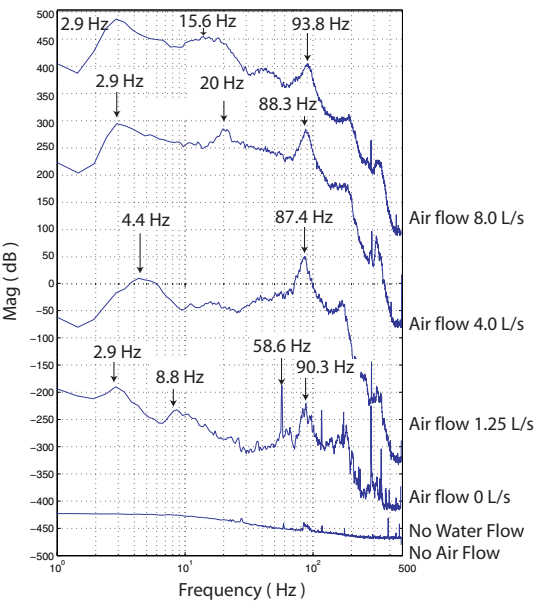

(b)

Figure 7: Power spectral density functions.

The dominant low frequency spikes were found to occur at identical frequencies for both reaction force and wall pressure spectra. As mentioned earlier, by examining high-speed video with reaction force and wall pressure time-history data sets, the low frequency energy was found to be attributed to the rotation rate of the liquid component in the SFE. It is believed that the dominant highfrequency spikes (approximately $70 \mathrm{~Hz}$ and $90 \mathrm{~Hz}$ for reaction force and wall pressure, respectively) are attributed to both mechanical vibrations and pressure fluctuations caused by the liquid pump. This claim is made based on the fact that these spikes only appear when the pump is running, and do not seem to change significantly with changing air flow rate. 
The dominant middle frequency spikes $(39.5 \mathrm{~Hz}, 31.3 \mathrm{~Hz}, 24.4 \mathrm{~Hz}$ for reaction force; $20 \mathrm{~Hz}, 15.6 \mathrm{~Hz}$ for wall pressure) are believed to be attributed to the formation, convection, and exiting of the liquid slugs in the SFE. This was based on examining high-speed video with its associated time-history data set in conjunction with the 30 second time-history data set taken independent of the high-speed video. The middle frequency energy for each air flow rate was found to be slightly different between reaction force and wall pressure. The reason for this is believed to be due to the fact that reaction force is an integral measure of liquid being accelerated in the SFE, whereas wall pressure fluctuation is related to either pressure build-up in the SFE, or a change in air-core diameter at the location of the wall pressure port. Pressure build-up in the SFE is associated with a "necking" of the air-core diameter at the inlet, and with liquid slugs exiting the cylindrical cavity.

\section{Future work and conclusions}

From this preliminary investigation, it is clear that future work must be done in two areas. The first will be to collect high-speed video with different geometries of SFE design. The second area will include developing a simple analytical model relating the wall pressure and air-core pressure to the diameter of the air-core in the SFE. Conditions for the failure of this rudimentary model may connote a transition to the SF regime. In conclusion, an experimental apparatus suitable for analysis of a SFE was designed in the course of this investigation. The autocorrelation and crosscorrelation functions of pressure and reaction force signals aided in understanding the transition to the slug flow regime. Power spectral density functions of reaction and wall pressure for the SF regime were shown to have energy at low, middle, and high frequency components.

\section{References}

[1] C.E. Brennen. Fundamentals of Multiphase Flow. Cambridge University Press, 2005.

[2] R.C. Chanaud. Observations of oscillatory motion in certain swirling flows. Journal of Fluid Mechanics, 21:111127, 1965.

[3] R.J. Hugo and C. Veer. An experimental investigation of vortex breakdown in multiphase pipe flow. AIAA-2003-3602, 2003.

[4] H. Sato and K. Watanabe. Experimental study on the use of a vortex whistle as a flowmeter. IEEE Transactions on Instrumentation and Measurement, 49:200205, 1954.

[5] B. Vonnegut. A vortex whistle. Journal of the Acoustical Society of America, 26:1820, 1954.

[6] P.D. Welch. The use of fast fourier transform for the estimation of power spectra: A method based on time averaging over short, modified periodograms. IEEE Trans. Audio Electroacoust., pages 7073, 1967.

[7] Z. Zhang and R. J. Hugo. Stereo particle image velocimetry applied to a vortex pipe flow. Experiments in Fluids, 40, 2006. 